\title{
Three-dimensional Reconstruction and Visualization of the Cerebral Cortex in Primates
}

\author{
Sergio Demelio ${ }^{1}$, Fabio Bettio ${ }^{1}$, Enrico Gobbetti ${ }^{1}$, and Giuseppe Luppino ${ }^{2}$ \\ 1 CRS4, VI Str. Ovest, Z.I. Macchiareddu, C.P. 94, I-09010 Uta (CA), Italy \\ http: //www.crs4.it/vvr \\ \{sergio, fabio, gobbetti\}ecrs4.it \\ 2 Institute of Human Physiology, University of Parma, I-43100 Parma, Italy \\ luppino@ipruniv.cce.unipr.it
}

\begin{abstract}
We present a prototype interactive application for the direct analysis in three dimensions of the cerebral cortex in primates. The paper provides an overview of the current prototype system and presents the techniques used for reconstructing the cortex shape from data derived from histological sections as well as for rendering it at interactive rates. Results are evaluated by discussing the analysis of the right hemisphere of the brain of a macaque monkey used for neuroanatomical tract-tracing experiments.
\end{abstract}

\section{Introduction}

One major field of interest in neuroscience is the study of the anatomical and functional organization of the cerebral cortex in primates. One widely accepted notion is that cortical information processing occurs in parallel, along different specialized cortical circuits, linking anatomical and functional cortical units, generally referred to as cortical areas (see, e.g., [7]). One major challenge is thus, at present, the definition of the exact number, extent and functional properties of the various cortical areas. Non invasive modern functional imaging techniques (PET, fMRI) allow addressing this issue in human subjects. These techniques, however, have a very poor temporal and a low spatial resolution, limited to the macrostructural level. On the other hand, a variety of more invasive methodological approaches may be used in experimental animals and, in this line of research, non human primates represent the experimental model closest to the human brain. These approaches allow acquisition of data with high spatial and temporal detail, so that the brain of non human primates is unanimously considered a crucial anatomical and functional frame of reference for studies on human subjects.

Basically, three main categories of experimental approaches may be used in non human primates, possibly combined together in the same animal: i) the morphological approach is based on the study of the regional variability of the cortical structure and is aimed to define the anatomical borders between different cortical areas in individual brains; given the interindividual variability no standard brain atlas may be used as anatomical frame of reference; ii) the anatomical approach is based on microinjections of substances transported by the neurons (neural tracers); the qualitative and quantitative distribution of neurons labeled with the tracers employed, allows the definition of the connections of a given cortical area with other cortical areas or subcortical 
structures; iii) the electrophysiological approach is based on the recording with microelectrodes of the electrical activity of single neurons in different behavioral tasks and therefore, on the definition of their functional properties.

Each of these experimental procedures requires the analysis of the collected data at the microstructural level. Basically, the brain is cut in serial sections (thickness about $50 \mu \mathrm{m}$ ), oriented so as to have an optimal view of the region of interest. After appropriate histological processing, sections are mounted on slides and individually analyzed with low or high power microscopy. Typically, this step of the analysis relies on the manual selections in each sampled section of different sets of points, grouped in different classes, that represent the $\mathrm{X}-\mathrm{Y}$ coordinates of the various data of interest. These data always include the outer and the inner border of the cortical mantle and may include the locations of borders between areas, locations of individual neurons labelled with each of neural tracers employed (see, e.g., figure 1) or the location of the tracks of microelectrode penetrations.

The typical final step in the analysis and interpretation of the data is, then, the analysis of static images of brain sections or, for an overall view of the collected data, of the $2 \mathrm{D}$ reconstruction of the cortical surface, made by flattening and aligning the cortical contours of the sampled sections [3]. However, the procedure of flattening a surface with a very complex geometry, due to the presence of deep cortical sulci with irregular shapes and spatial arrangement (see, e.g., figure 1), is an unavoidable source of distortions that can seriously affect a correct interpretation of the data. This problem becomes even greater in the comparison of data obtained in different animals in which, in order to have an optimal view of the regions of interest, brains are cut with different sectioning planes. In this case, sections and flattened views of the same region of brains cut with different angles can be very difficult to compare, since researchers are forced to mentally construct a model of 3D shapes, adding further complexity to what is an already challenging task. Given these problems, 3D reconstruction and, therefore, the creation of a virtual environment for the direct analysis in three dimensions of the cortical structure, would be of crucial value in the interpretation of these types of data.

In the remainder of this paper, we provide an overview of our prototype system and discuss experimental results obtained on the right hemisphere of the brain of a macaque monkey used for neuroanatomical tract-tracing experiments.

\section{Methods and tools}

Our interactive system takes as input data acquired by identifying contours and neuron positions on cryogenized brain sections and regards it as a three-dimensional environment to be interactively inspected.

The full potential of 3D rendering is harnessed when allowing operators to view and naturally interact in real-time with the reconstructed 3D data. In particular, the ability to render at interactive speeds dramatically improves depth perception, thanks to motion parallax effects. To support the required interactive performance, we have chosen to exploit the capabilities of current polygon-oriented graphics accelerators by first reconstructing the cortex boundary surfaces and then rendering them, using a multipass technique to also incorporate in the same image subsurface neurons. As illustrated in 


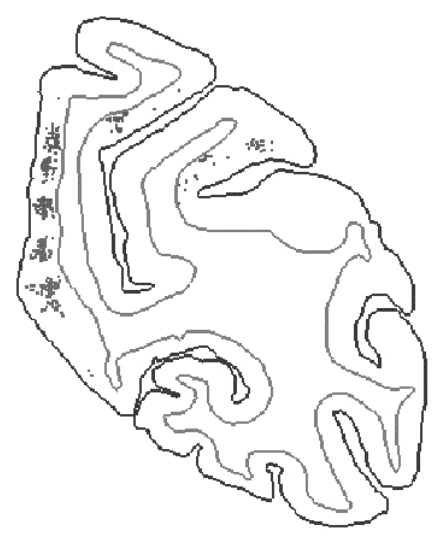

Fig. 1: Typical brain section. We can see the external and internal cortical lines, as well as different groups of neuronal cells

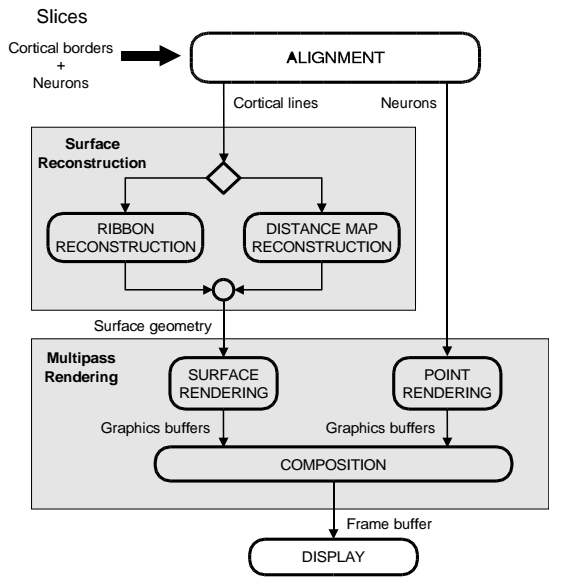

Fig. 2: System overview. Alignment and surface reconstruction are performed once for each data set in a pre-processing phase, while the the multipass rendering algorithm is embodied in a simple prototype analysis tool.

figure 2, the main operations performed by the system are thus section alignment, to position all data in the same frame of reference, surface reconstruction, to construct, using different algorithms, triangular meshes that approximate the cortex boundaries, and multipass rendering, to display at interactive rates images of the cortical surface and of subsurface neurons. Section alignment and surface reconstruction are performed once for each dataset in a preprocessing phase, while the multipass rendering algorithm is embodied in a prototype analysis tool that offers a simple user interface for performing standard viewing and inspection operations, including camera motion, control of rendering parameters (e.g. transparency), and of cutting planes.

The prototype has been developed with GLUT/OpenGL and is running both on Unix and Windows platform. We have implemented and tested it on a Silicon Graphics Onyx InfiniteReality, with 2 MIPS R10000 $194 \mathrm{MHz}$ CPU, and on a PC equipped with two PIII $600 \mathrm{MHz}$ CPU and a GPU NVIDIA GeForce2. The results obtained on the PC have been comparable (and often superior!) to those obtained on the Onyx.

Details on each of the main operations are provided in the following sections.

\subsection{Section alignment}

In order to correctly reconstruct the brain, the different sections have to be aligned according to the same frame of reference. A variety of manual, semi-automatic, or automatic techniques have been presented in the literature. In our case, the system has to work without the use of fiducial markers, and the process is made more complex 
because of the possible small-scale deformations (e.g. lobe motions) caused by the particular acquisition process. For this reason, we have adopted a simple manual retrospective technique in which the operator directly defines the parameters of the registration transformation by interactively positioning one section with respect to the other, relying on his judgment of the relative location of specific anatomical features.

While the process is time-consuming, and limited by the precision with which the operator can provide alignment information, we consider the solution practical enough for laboratory work, especially since reconstructing a given brain requires manual alignment of only about seventy sections. Moreover, it is unlikely that totally automatic techniques, such as those successfully used for data fusion applications (e.g. for registering images of the same anatomy acquired in different modalities), are applicable without manual intervention in our case, since semantic ability is required to "fill the gaps" between neighboring sections.

The major current limitation is coming from the constraint that we currently support only rigid transforms. We are currently considering the introduction of global stretching and second degree polynomial transforms (as in [1]) and of local transformations for correcting lobe displacements.

\subsection{Surface reconstruction from aligned cross-sectional data}

The goal of surface reconstruction is to to generate, from the set of contours present in the aligned brain sections, a triangular mesh of the cortex surfaces. Obtaining a triangular mesh for each boundary surface is extremely useful for high speed rendering on current graphics hardware.

A number of authors have presented techniques for reconstructing surfaces from the cross-sections of an object. All techniques can be classified in two distinct approaches: direct triangulation or shape based functional techniques [12]. The first approach directly triangulates the set of points making up each of these cross-sections, such that they become the vertices of the triangular mesh. While the technique is conceptually simple, many problems arise when the cross-sectional shape varies widely between planes (e.g. holes, branching structures), and much effort is required to detect and correct special cases where the triangulation of complex shapes might otherwise fail [10, 11]. The second approach is to estimate from the contours in the cross sections a 3D function, discretized on a grid, which represents the distance at any point in space to the nearest boundary surface. Numerous algorithms have been proposed to efficiently compute such a distance map (an excellent survey is provided in [5]). Once the function has been created, the iso-surface can be triangulated by a variety of algorithms, including marching cubes $[13,6]$.

It is important to note that it is intrinsically impossible to guarantee that a particular technique will reconstruct the actual anatomy from a particular set of cross-sections. For this reason, we have chosen to complement the reconstruction using a distance map technique with a discontinuous method which duplicates the profile of a contour along the thickness of the section, so that the representation we obtain is a surface made up of "ribbons". The second method makes it possible to evaluate the relative positioning of the different sections and judge possible misalignment problems. Figure 4 shows a 
typical exterior cortex surface reconstructed with both methods, which are described in more detail below.

Ribbon surface reconstruction The goal of the algorithm is to create well formed surfaces that represent the boundary of the solid constructed by stacking all the sections one on top of the other. For each of the boundaries, a triangular mesh is constructed using the following technique:

1. for each section, the contour is duplicated in the vertical direction at the distance given by the section thickness; the resulting strip is triangulated and the triangles are added to the mesh;

2. the gaps between adjacent sections are closed by triangulating the planar regions delimited by the boundaries of the sections (see figure 3); this can be efficiently done the following way:

(a) the intersection points between two subsequent contours are computed (these are always in even number since the contours are closed);

(b) the closed contours of each of the regions included between two subsequent intersection points are reconstructed;

(c) a Delaunay triangulation of each new computed regions is carried out, and the computed triangles are added to the mesh.

The obtained reconstruction presents the advantage of not altering the initial dataset, and, while it is a quite rough representation of the most likely smooth surface, it still provides a good impression of the overall brain shape.

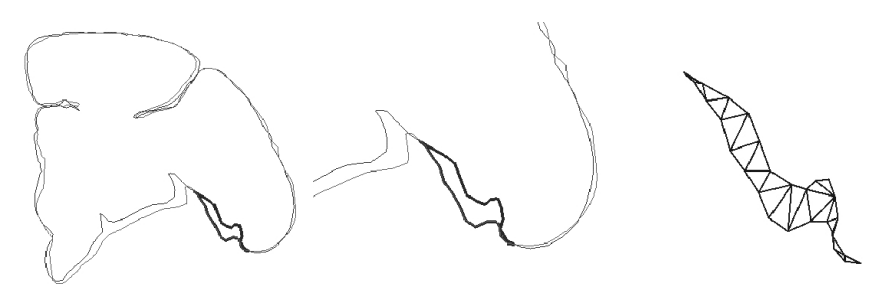

Fig. 3: "Ribbon" surface reconstruction. From the left: a pair of adjacent sections; detail of a region delimited by two points of intersection; detail of the region triangulation.

Distance map surface reconstruction The goal of the distance map algorithm is to construct a triangular mesh for each boundary surface that is a likely piecewise linear approximation of the corresponding real cortical boundary. Smoothed images can then be obtained at interactive speeds by exploiting the Gouraud shading algorithm. In our implementation, the reconstruction algorithm performs the following passes: 
1. a planar distance function is created for every section; this distance transformation maps a binary image (consisting of boundary pixels on a zero background) into an image where all corresponding background pixels have a value proportional to the distance to the nearest boundary pixel. This mapping can be created analytically, but a global operation like this is prohibitively costly [4]. A more efficient way of calculating a distance transform is through a chamfer coding [2], which allows good approximations of the Euclidean distance with small masks applied sequentially on local neighborhoods. For our computations, we have used the (5:7:11) $3 \times 5$ chamfer mask;

2. a spatial distance function is created for the entire volume; the best results can be obtained by interpolating according to suitable directions among adjacent plane distance functions [13], in order to take into account the variations in the topology of the surface. In our case, the sections are not very sparse, and a 3D distance function obtained without interpolation has also provided good results;

3. the zero isosurface is extracted: the method applied is a marching cube algorithm [6].
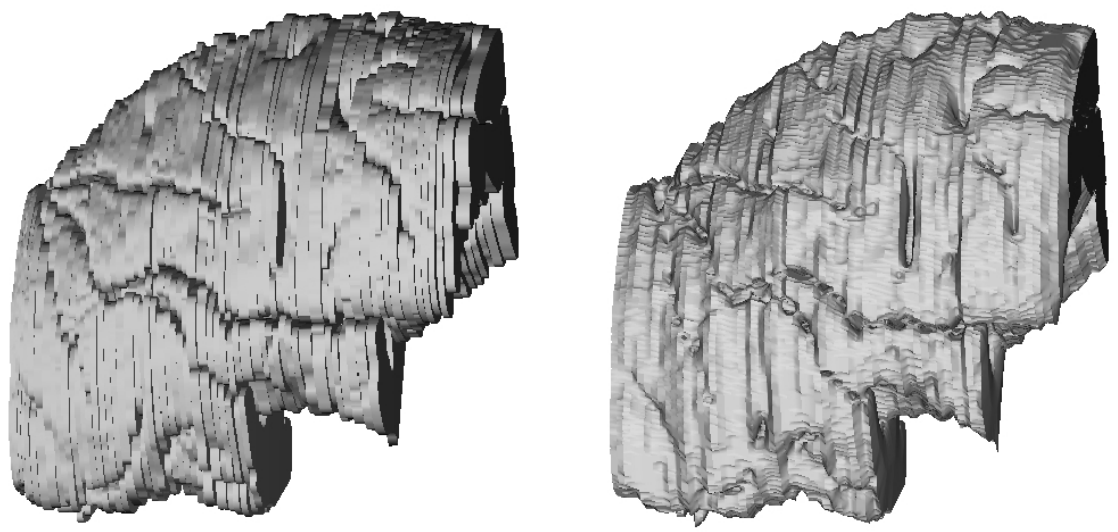

Fig. 4: Surface reconstruction. In the left, the cortex surface reconstructed with the "ribbon" method; in the right, the same surface reconstructed with the distance map method. The distance map surface is more "readable" because of the more likely distribution of curvatures.

\subsection{Interactive rendering}

The rendering system needs to present at interactive rates both surface elements (i.e. the cortex) and subsurface elements (i.e. the neurons). In order to present in the same image details of the interior of the brain that convey depth information, a volumetric approach has to be taken. To obtain interactive performance, we have decided to exploit 
the power of current graphics boards to accelerate rendering and compositing tasks. The implementation of advanced rendering algorithms using multipass techniques is becoming a standard approach in interactive applications, motivated by the increasing performance of graphics chips. Many special- or general-purpose techniques have been presented in the literature. An excellent survey is provided in [9].

In our case, we model neurons as emissive particles and the cortex material as an absorbing (semi-transparent) medium with constant absorption coefficient. Since the position of a given neuron is only known in its section's plane, we apply to each neuron a random shift in the thickness direction, to obtain a more likely neuron distribution. Rendering, using orthographic projections, is then performed using the following passes:

1. the cortical surface is rendered using lighting and Gouraud shading;

2. the Z-buffer and the color buffer are copied into memory;

3. neurons are rendered with $Z$-test enabled and no lighting; each neuron is assigned a color ID which identifies its type;

4. the Z-buffer and the color buffer are copied into memory;

5. the color buffer is updated by computing neuron colors as a function of their ID and of the difference between the two Z-buffer values;

6. the updated color buffer is written to frame memory for display; a convolution filter may be applied to perform image smoothing operations.

The attenuation computed by the algorithm is exact, to the limits of the Z-buffer precision, for convex surfaces, but is possibly overestimated for concave boundaries, since multiple intersections of the eye-neuron ray with boundary surfaces are ignored (see figure 5 left). We consider this error negligible for all practical applications, since the only visible neurons are those close to the exterior surface and the brain has a particular shape with very thin sulci. Figure 5 middle and right illustrate the effects of the emission-absorption model.
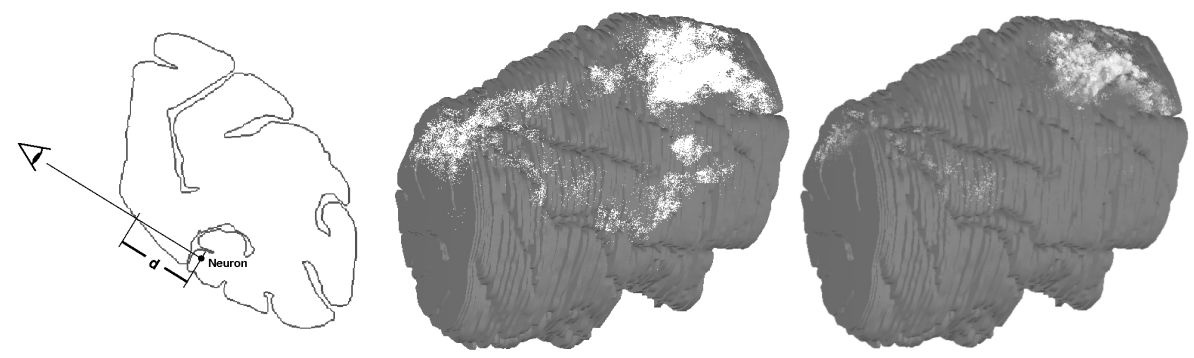

Fig. 5: Neuron rendering. Neurons are modeled as emissive particles, while the brain material is considered a semi-transparent medium with constant absorption coefficient. Absorption is computed based on the distance from the neuron to the surface closest to the eye ( $d$ in the left image). In the middle, neurons are rendered all with the same intensity. In the right, their color is attenuated by a factor which depends on the distance to the surface in the viewing direction, effectively providing depth information 


\section{Case study}

Our system has been tested on medical datasets acquired at the Institute of Human Physiology of the University of Parma. The case discussed in this report is the right hemisphere of the brain of a macaque monkey used for neuroanatomical tract-tracing experiments. This experiment was made in the framework of a line of research devoted to the exact definition of the source of cortical projections to each of the various anatomical and functional areas that form the motor cortex in the monkey [8]. These data are of extreme importance for the interpretation of the possible functional role in motor control of each motor area and for the definition of the various cortical circuits involved in different types of information processing for voluntary action. In this particular case, in compliance with Italian and European laws on the care and use of laboratory animals, under aseptic condition and under microscopic guidance, three restricted injections of different fluorescent neural tracers (FB, DY, TB) were made in the two areas that form the so called "dorsal premotor cortex". After appropriate survival period the animal was sacrificed and the brain cut frozen in serial sections $(60 \mu \mathrm{m}$ thickness) in coronal plane. Under fluorescence microscopy each of the three fluorescent tracers emits light of different color so that neurons labeled with each tracer (and, therefore, neurons that are anatomically connected with the injected cortical site) can be easily identified at medium-power enlargement (200-400X). In one section each $600 \mu \mathrm{m}$ the outer and inner cortical contours were manually delineated and saved as different classes of points along with the location of individual labeled neurons plotted and grouped in three different classes of points according to the type of tracer for which they were positive. Figure 1 is an example of data collected from one individual brain section.

The source dataset is made up of about 70 sections that cover the whole extent of the brain regions labeled with the tracers. The number of points acquired in each section

is about $10^{3}$ per boundary, which means that the $3 \mathrm{D}$ model contains over $10^{5}$ vertices. Figures 6 and 7 (color plates) show the results of the reconstruction procedure. In Figure 6 (left) is shown a lateral view of the hemisphere in which the frontal lobe is on the right and the occipital lobe on the left. As one can see from the figure, the result is very realistic. All the major cortical sulci are clearly distinguishable and the same is true for finer macroscopical details as for example small cortical dimples. Furthermore, the possibility of interactively rotating the hemisphere and zooming on regions of particular interest allows operators to have a closer view of these macroscopic details and to benefit from parallax effects that provide additional depth cues.

It is quite clear, therefore, that this procedure appears to be effective in producing a reliable 3D reconstruction of individual brains. In this respect, one point that needs to be stressed is that the reliability of reconstructions of individual brains is extremely helpful when results from different experimental cases are compared. In fact, interindividual variability in the gross morphology may account for apparent differences among different animals.

One problem faced in the alignment of the sections is represented by some discontinuities between profiles of adjacent sections. These discontinuities are actually due to some deformations of the sections that eventually occur during the histological processing or when mounted on slides. This problem can be overcome by improved alignment techniques (see section 2.1) or by an appropriate intervention before align- 
ment. In the same figure is also shown the distribution of the labeled cortical neurons. Neurons labeled with different tracers are shown with dots of different colors and the color intensity is attenuated depending on the distance of the neurons from the surface in the viewing direction. In the upper part of the frontal lobe three densely aggregate of labeling surround the injections of the three tracers. The injected region can be better appreciated in Figure 7 that represents a dorsal view of the hemisphere. Because of the large and concentrated number of labeled neurons in these regions the dots appear to be fused together. However, when the region is zoomed in (Figure 7 right) the three clouds of labeling can be much better resolved. The sites of the injections are here provisionally represented as the regions empty of labeling within each of the three clouds of labeling. It is quite clear that this reconstruction procedure and appropriate reslicing could be a very powerful tool for the exact definition of the location and extent of the cortical region interested by the tracer injection. The differential distribution of the labeling in the various cortical region is clearly visible in all the figures presented. These presentations confirm, but also extend previous observations made in this case with conventional bidimensional flattening of the cortical surface. For example in the lateral view of the hemisphere, shown in Figure 6, left, it very clear the differential distribution of the labeling in the caudal part of the hemisphere, corresponding to the inferior parietal lobule. Although already noticed with conventional reconstruction techniques, the relative position of the positive neurons and their location relative to the sulci is now much clearer. The present reconstruction procedure appears therefore very helpful for the interpretation of the data and for the attribution of the labeling to particular cortical areas. Additional aspects that would be very difficult to appreciate with conventional bidimensional reconstruction can be clearly revealed by this procedure. For example, in the mesial view of the brain shown in the right part of Figure 6, it is possible to see, in the caudal part of the hemisphere, a differential distribution of various neuronal populations. Preliminary morphological data collected in the Institute of Human Physiology of the University of Parma indicate that several different anatomical areas form this region. Correlation of architectonic borders with the distribution of the labeling in this reconstruction would be a strong argument in favor of this hypothesis.

\section{Conclusions and future work}

We have presented a prototype interactive application for the direct analysis in three dimensions of primate cerebral structures. The system takes as input data acquired by identifying contours and neuron positions on cryogenized brain sections and regards it as a three-dimensional environment to be interactively inspected.

To support interactive performance, the system exploits the capabilities of current graphics accelerators by first reconstructing the cortex boundary surfaces and then rendering them, using a multipass technique to also show in the same image subsurface neurons.

While none of the techniques used are original from a graphics point of view, their combination in an interactive setting promises to be a valuable improvement over standard analysis tools used in neuroscience. In particular, the availability of low-cost, high performance graphics accelerators for PC platforms makes it possible to consider in- 
teractive $3 \mathrm{D}$ inspection of high resolution neuroanatomical data practical for everyday laboratory work. The system has been tested on actual datasets, and the feed back from end-users has been positive. In this work, we have discussed the inspection of the right hemisphere of the brain of a macaque monkey used for neuroanatomical tract-tracing experiments.

We are currently working on extending the system with improved reconstruction and visualization techniques. Particular areas of development are non-rigid section alignment and presentation of cytoarchitectural information about cortical tissue.

\section{Acknowledgments}

All the datasets used in the present work were supplied by the Institute of Human Physiology of the University of Parma. This work is partially funded by MURST under project: "Laboratorio Avanzato per la Progettazione e la Simulazione Assistita al Calcolatore". We also acknowledge the contribution of Sardinian regional authorities.

\section{References}

1. C. Bohm, T. Greitz, D. Kingsley, B. M. Berggren, and L. Olsson. Adjustable computerized stereotaxic brain atlas for transmission and emission tomography. American Journal of Neuroradiology, 4:731-733, 1983.

2. Gunilla Borgefors. Distance transformations in digital images. Computer Vision, Graphics, and Image Processing, 34(3):344-371, June 1986.

3. G. J. Carman, H. A.Drury, and D. C. van Essen. Computational methods for reconstructing and unfolding the cerebral cortex. Cerebral Cortex, 5:506-517, 1995.

4. O. Cuisenaire and B. Macq. Fast Euclidean distance transformation by propagation using multiple neighborhoods. Computer Vision and Image Understanding: CVIU, 76(2):163172, November 1999.

5. Olivier Cuisenaire. Distance Transformations: Fast Algorithms and Applications to Medical Image Processing. PhD thesis, Université Catholique de Louvain, 1999.

6. W. E. Lorensen and H. E. Cline. Marching cubes: a high resolution 3D surface construction algorithm. In M. C. Stone, editor, SIGGRAPH '87 Conference Proceedings (Anaheim, CA, July 27-31, 1987), pages 163-170. Computer Graphics, Volume 21, Number 4, July 1987.

7. G. Luppino and G. Rizzolatti. The organization of the frontal motor cortex. News in Physiological Sciences, 15:219-225, 2000.

8. M. Matelli, P. Govoni, C. Galletti, D.F. Kutz, and G. Luppino. Superior area 6 afferents from the superior parietal lobule in the macaque monkey. The Journal of Comparative Neurology, 402:327-352, 1998.

9. Tomas Moller and Eric Haines. Real-Time Rendering. A. K. Peters Limited, 1999.

10. J.-M. Oliva, M. Perrin, and S. Coquillart. 3D reconstruction of complex polyhedral shapes from contours using a simplified generalized voronoi diagram. Computer Graphics Forum, 15(3):307-408, August 1996. Proceedings of Eurographics '96. ISSN 1067-7055.

11. Bradley A. Payne and Arthur W. Toga. Surface mapping brain function on $3 \mathrm{D}$ models. IEEE Computer Graphics and Applications, 10(5):33-41, September 1990.

12. S. P. Raya and J. K. Udupa. Shape-based interpolation of multidimensional objects. IEEE Transactions On Medical Imaging, 9(1):32-43, March 1990.

13. G. M. Treece, R. W. Prager, A. H. Gee, and L. Berman. Surface interpolation for sparse cross sections using region correspondence. In Medical Image Understanding and Analysis 1999, July 1999. 

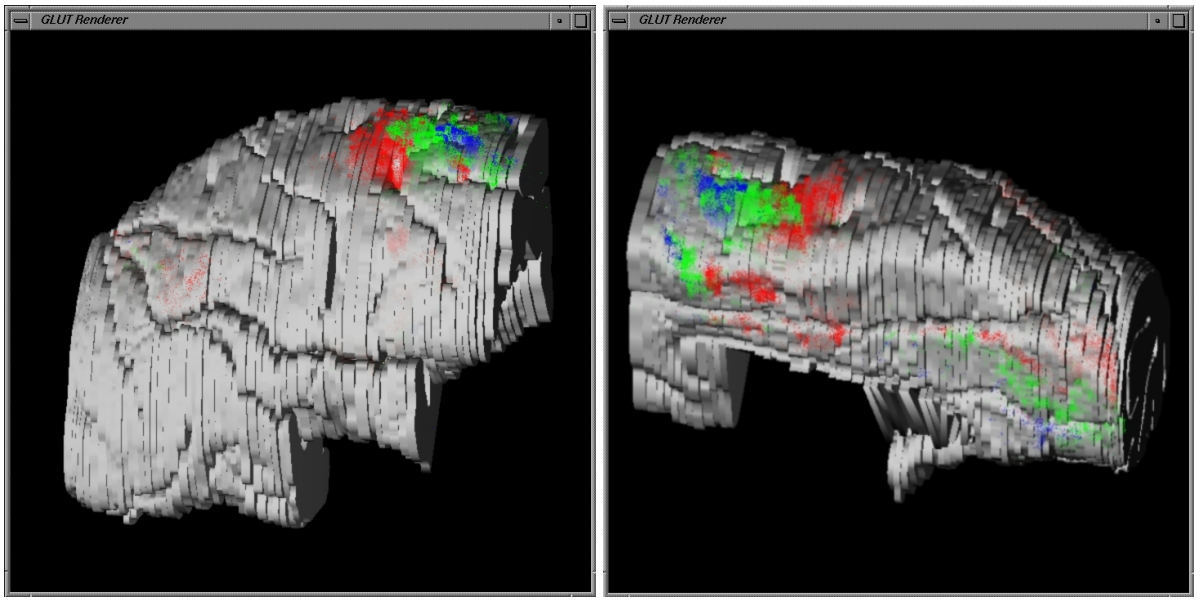

Fig. 6: [COLOR PLATE] Lateral (left) and mesial (right) view of the right hemisphere of the brain of a macaque monkey. All the major cortical sulci are clearly distinguishable and the same is true for finer macroscopical details as for example small cortical dimples. Neurons labeled with different tracers are shown with dots of different colors and the color intensity is attenuated depending of the distance of the neurons from the surface in the viewing direction.
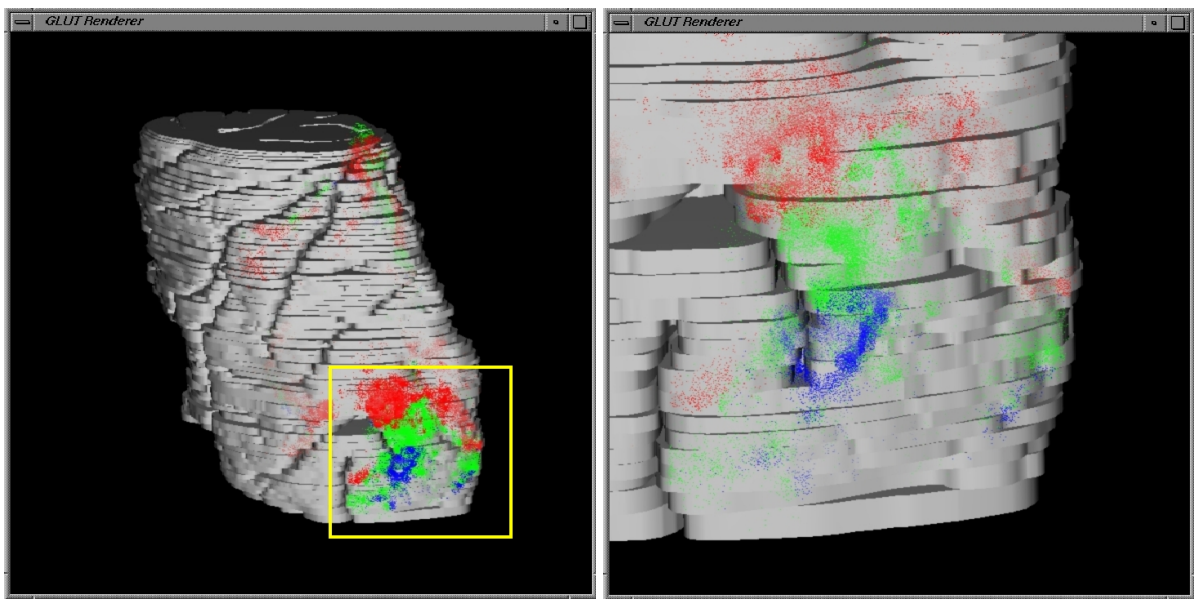

Fig. 7: [COLOR PLATE] Dorsal view of the right hemisphere of the brain of a macaque monkey. Three densely aggregates of labeling surround the injections of three neural tracers. The sites of the injections are clearly identified in the zoomed image as the regions empty of labeling within each of the three clouds of labeling. 